\title{
Contraintes résiduelles dans des éprouvettes cylindriques
}

\section{P. BÉREST}

Laboratoire de mécanique

des solides

École polytechnique

91128 Palaiseau Cedex berest@lms.polytechnique.fr

\section{G. VOUILLE}

Centre de Géotechnique et Exploitation du sous-sol École nationale supérieure des mines de Paris

35, rue Saint-Honoré 77305 Fontainebleau Cedex vouille@cges.ensmp.fr
On attribue fréquemment aux « contraintes résiduelles » ou ( contraintes internes ) diverses manifestations anormales observées dans les massifs rocheux ou sur des carottes prélevées en profondeur. Pour décrire ces phénomènes on considère une loi de comportement élastique linéaire présentant des « déformations initiales » non compatibles. Les contraintes résiduelles ne dépendent alors que de la forme de l'objet considéré, des paramètres élastiques et du tenseur d'incompatibilité (ou tenseur de Ricci) associé aux déformations initiales. En supposant que ce tenseur est constant dans une petite éprouvette cylindrique prélevée dans le massif rocheux, on établit la forme générale des champs de contrainte résiduelle qui peuvent affecter l'éprouvette.

Mots clés : contraintes résiduelles, incompatibilité des déformations.

\section{Residual stresses in cylindrical samples}

Several (canomalous phenomena ) observed in rock masses or rock samples are attributed to the so-called « residual stresses» or (internal stresses». These phenomena can be simply described by assuming linear elastic behavior and incompatible " (initial » strains. Then residual stresses distribution only depends upon the shape of the considered body, the value of the elastic parameters and the incompatibility (or Ricci) tensor which is associated to the initial strains tensor. It is reasonable to assume that the incompatibility tensor is constant in a small cylinder sampled out from the rock mass; then the distribution of residual stresses in the sample can easily be computed.

Key words : residual stresses, strains incompatibility. 


\section{Introduction}

On appelle « contraintes résiduelles ) les contraintes qui se développent dans un corps soumis à un chargement extérieur nul (Cornet, 1993). Suivant les contextes, on parle aussi de (contraintes internes») ou ( contraintes de croissance » (dans le cas du bois, voir Archer, 1986). Elles sont le signe de l'absence d'un ( état naturel ) dans lequel les contraintes seraient identiquement nulles dans tout domaine non chargé.

On attribue aux contraintes résiduelles de nombreuses manifestations " anormales » observées en mécanique des roches : convergence excessive dans un tunnel, rupture sous chargement mécanique apparemment modéré en comparaison de la résistance du matériau, disquage des carottes prélevées en profondeur, etc.

Comme pour tous les phénomènes mal connus, il est possible qu'on prête aux contraintes résiduelles préexistantes des effets qui relèvent en fait d'autres origines (effets de la température, de l’hygrométrie, qui engendrent des contraintes résiduelles; mais aussi contraintes d'origine tectonique, effet du travail de l'outil de coupe...). Il est certain en tout cas que l'existence de contraintes résiduelles peut conduire à une interprétation incorrecte des résultats de nombreuses mesures classiques (convergence dans un tunnel, détermination de l'état de contraintes par surcarottage, détermination de l'enveloppe de rupture).

On peut obtenir des contraintes ( internes ) dans une structure en assemblant plusieurs éléments, dont chacun séparément présente un état naturel, mais dont les déplacements aux points ou surfaces d'assemblage ne sont pas ( compatibles ». On crée par exemple des contraintes en élevant la température d'un assemblage hyperstatique de barres articulées dont le coefficient de dilatation thermique n'est pas le même pour chaque barre.

On s'intéresse plutôt dans cet article à des matériaux qui ne présentent pas d'état naturel même lorsqu'on en considère une partie arbitrairement petite (mais de volume non nul). L'évaluation des contraintes résiduelles dans ce contexte est classique dans le cas des métaux : on peut mesurer les déformations résiduelles à la surface d'une pièce en utilisant les rayons X. On effectue plusieurs mesures en modifiant la surface par enlèvement de matière ; mais comme on modifie ainsi les contraintes résiduelles, qui sont toujours relatives à la forme de l'objet considéré, la reconstruction du champ de contraintes dans la pièce initiale est délicate (Ballard et Constantinescu, 1994). Dans le cas du bois on utilise la méthode du ( trou unique ». En mécanique des roches, Hardy et Mangolds (1980) ont proposé des techniques de mesure dans le cas du sel gemme.

Le présent article vise à présenter les équations permettant de déterminer les contraintes résiduelles. Après quelques rappels de définitions classiques, on introduit le tenseur d'incompatibilité, qui est le tenseur de Ricci associé en chaque point au tenseur des déformations initiales (obtenu en chaque point en annulant le tenseur des contraintes dans la loi de comportement). Dans le cas d'un comportement élastique, les contrainte résiduelles ne dépendent que du domaine considéré, des paramètres élastiques et du champ de tenseur d'incompatibilité. Supposant ce dernier constant dans un cylindre de roches prélevé en pro- fondeur, on met en évidence deux distributions possibles du tenseur des contraintes résiduelles dont toutes les autres se déduisent par combinaison linéaire.

\section{2 \\ Définitions}

\section{1}

\section{Champ d'autocontraintes}

Un champ de tenseurs $\underline{\sigma}=\underline{\sigma}(\underline{x})$ symétriques défini dans un domaine matériel $\bar{\Omega}$ est dit champ d'autocontraintes si les conditions suivantes sont vérifiées :

$\begin{array}{ll}\operatorname{div} \underline{\underline{\sigma}}=0 & \text { dans } \Omega \\ \underline{\sigma} \cdot \underline{\underline{n}}=\underline{0} & \text { sur } \partial \Omega \\ {[[\underline{\sigma}]] \cdot \underline{N}=\underline{0}} & \text { dans } \Gamma\end{array}$

On dit alors que le domaine $\Omega$ est (non chargé ).

Les deux premières relations indiquent que le corps n'est soumis ni à des forces de volume, ni à des forces de surface sur son contour extérieur $\partial \Omega$. La troisième relation indique que $\underline{\sigma}$ peut être discontinu à la traversée d'une frontière interne $\Gamma$, mais que le vecteur contrainte normal à la discontinuité doit être continu.

Pour un domaine $\Omega$ donné, il existe une infinité de champs d'autocontraintes. Ce n'est que lorsque la relation de comportement du matériau constituant $\Omega$ a été précisée qu'on peut déterminer le (parfois les) champ(s) d'autocontraintes. Par exemple dans le cas du comportement élastique sans déformations initiales le seul champ d'autocontraintes est $\underline{\underline{\sigma}}=\underline{\underline{0}}$.

\section{2}

\section{Conditions de compatibilité et de fermeture}

On note $\xi=\xi(x)$ le champ de déplacement compté depuis une configuration de référence; dans l'hypothèse des petites perturbations, le tenseur des déformations est noté :

$$
\stackrel{\varepsilon(x)}{=}=\frac{1}{2}\left(\underline{\underline{\nabla \xi}}+{ }^{\tau} \underline{\underline{\nabla \xi}}\right)
$$

Lorsqu'on se donne inversement un champ de tenseurs $\underline{\underline{\varepsilon}}=\underline{\underline{\varepsilon}}(\mathrm{x})$ symétrique, on peut se demander à quelles conditions il est ( un champ de déformation »), c'est-à-dire s'il existe un champ de déplacement $\underline{\xi}=\underline{\xi}$ (x) vérifiant l'égalité (2) quand on n'impose aucune autre sujétion au champ $\xi=\underline{\xi}(\mathrm{x})$. Deux conditions doivent être vérifiées:

\section{a. Conditions de compatibilité}

On peut trouver un champ de déplacement $\underline{\xi}=\underline{\xi}(\underline{x})$ vérifiant (2), où $\underline{\varepsilon}=\underline{\varepsilon}(\mathrm{x})$ est donné si, de manière équijvalente, l'un ou l'autre des deux tenseurs suivants est nul :

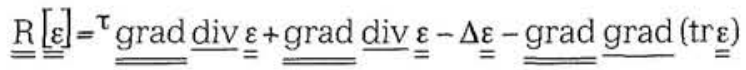

$$
\begin{aligned}
& \underline{\underline{E}}[\varepsilon]=\underline{\underline{R}}-1 / 2(\operatorname{tr} \underline{\underline{R}}) \stackrel{1=}{=} \underline{\underline{\operatorname{rot}}} \mathrm{d} \stackrel{\text { rot }}{\underline{\underline{\varepsilon}}} \stackrel{\varepsilon}{=}
\end{aligned}
$$

Le premier tenseur, ou tenseur d'incompatibilité, est appelé « tenseur de Ricci (linéarisé) ) dans le contexte 
de la géométrie non euclidienne ; le second tenseur est dit (c tenseur d'Einstein ». Les deux tenseurs sont symétriques; de plus on a div $\underline{\underline{E}}=\underline{0}$ soit $\operatorname{div} \underline{\underline{R}}=\underline{\operatorname{grad}}(\operatorname{tr} \underline{\underline{\mathrm{R}}}) / 2$.

\section{b. Conditions de fermeture}

Si les conditions de compatibilité $(\underline{\underline{R}}=\underline{\underline{0}}$ ou $\underline{\underline{E}}=\underline{\underline{0}})$ sont satisfaites, on peut calculer un déplacement $\xi=\xi$ (x) à une isométrie près. Toutefois, lorsque le domaine d'intégration n'est pas simplement connexe (il contient des ( trous ») l'isométrie peut dépendre du chemin d'intégration; seule convient alors une solution pour laquelle le champ $\underline{\xi}=\underline{\xi}(\underline{x})$ est défini de façon univoque en chaque point du domaine.

\section{3}

\section{Champ de déformations initiales}

On considère dans la suite des relations de comportement de la forme $\underline{\varepsilon}=F(\underline{\sigma})$ telles que $\underline{\varepsilon}_{0}=F(\underline{0})$ ne soit pas nul; $\underline{\varepsilon}_{0}$ est dit champ de " déformations initiales ». Cette appellation est un peu impropre car on ne supposera pas en général que $\underline{\underline{\varepsilon}}_{0}$ soit compatible ; on notera $\underline{\underline{R}}_{0}=\underline{\underline{R}}$ $\left\{\underline{\varepsilon}_{0}\right\}$ son (tenseur d'incompatibilité ». Lorsque $\underline{\underline{R}}_{0}=\underline{R}_{0}(\mathrm{x})$ symétrique non nul est donné a priori, il ne peut constituer un tenseur d'incompatibilité que si la condition : $2 \operatorname{div} \underline{\underline{R}}_{0}=\operatorname{grad}\left(\operatorname{tr} \underline{\underline{R}}_{0}\right)$ est satisfaite. On peut alors calculer $\underline{\varepsilon}_{0}=\underline{\varepsilon}_{0}(\mathrm{x}) \overline{\overline{\text { dont }}} \underline{\underline{R}}_{0}$ est le tenseur d'incompatibilité ; mais $\underline{\varepsilon}_{0}$ ne sera défini qu'à un champ de déformation compatible près, puisque pour tout champ $\underline{\underline{\varepsilon}}=\underline{\underline{\varepsilon}}(\underline{\mathrm{x}})$ compatible :

$$
\underline{\underline{R}}\{\underline{\underline{\varepsilon}} 0-\underline{\underline{\varepsilon}}\}=\underline{\underline{R}}\{\underline{\underline{\varepsilon}} 0\}
$$

\section{4}

\section{État naturel}

On dit qu'un matériau présente un état naturel si, au moins pour un volume $\Omega$ assez petit, le seul champ d'autocontraintes est $\underline{\sigma}(\underline{x})=\underline{0}$. Il est alors commode de choisir cet état non contraint comme configuration initiale dite ( état naturel ». C'est possible par exemple dans l'hypothèse du comportement élastique linéaire isotrope :

$$
\underline{\underline{\varepsilon}}=\frac{1+v}{E} \stackrel{\underline{\sigma}}{=}-\frac{v}{E}(\operatorname{tr} \underline{\underline{\sigma}}) \underline{\underline{1}}
$$

Une autre définition de l'état naturel, peut être plus exploitable pratiquement, consiste à dire que si on coupe $\Omega$, non chargé, en deux domaines $\Omega_{1}$ et $\Omega_{2}$, non chargés, on n'engendre aucune déformation dans chacun des domaines créés.

\section{3}

\section{État non naturel}

\section{1}

\section{Définition et propriétés}

Lorsqu'un matériau ne présente pas d'état naturel, le champ d'autocontraintes réalisé dans un domaine $\Omega$ non chargé est non nul ; il est dit champ de contraintes résiduelles. Les contraintes résiduelles dépendent de manière essentielle du domaine $\Omega$ considéré : on change en général leur valeur en un point lorsque le domaine $\Omega$ non chargé contenant ce point varie. On peut de même qualifier les déformations réalisées dans ce contexte de ( résiduelles »; toutefois leur définition exige que soit précisée une configuration de référence, dont le choix est souvent arbitraire du fait même de l'absence d'un état naturel.

\section{2}

\section{Exemple de matériau sans état naturel}

On considère la relation de comportement suivante :

$$
\stackrel{\varepsilon}{=}=\underline{\underline{\varepsilon}} 0+\frac{1+v}{\mathrm{E}} \underline{\underline{\sigma}}-\frac{v}{\mathrm{E}}(\operatorname{tr} \underline{\underline{\sigma}}) \underline{1}
$$

où $\underline{\varepsilon}_{0}=\underline{\varepsilon}_{0}(\underline{\mathrm{x}})$ est un champ de tenseurs symétriques suffisamment régulier (deux fois dérivables pour fixer les idées; le cas d'un champ moins régulier est évoqué plus loin). Ce champ est dit « de déformations initiales »; il ne s'agit pas nécessairement d'un champ compatible.

Une telle relation apparaît naturellement dans de nombreux modèles de comportement usuels. Par exemple dans le modèle thermoélastique on a $\underline{\underline{\varepsilon}}_{0}=\alpha \mathrm{T} \underline{\underline{1}}$, où $\alpha$ est le coefficient de dilatation thermique et T l'écart de température depuis un état naturel. Le modèle élastoplastique, ou les modèles élastiques avec apparition de retrait, gonflement, changement de phase, fournissent aussi des relations de la forme (6).

Dans la suite on ne se préoccupe pas de l'origine physique de la déformation initiale ni de l'histoire des transformations qui ont conduit à l'apparition de cette déformation initiale.

La loi retenue est très simple ; il est entendu que pour un matériau concret, elle pourrait être remplacée par une loi de comportement anisotrope, hétérogène, ou non linéaire notamment si le domaine de variation des contraintes envisagé est étendu.

\section{3}

\section{Propriétés du matériau}

Si la déformation initiale est compatible, $\underline{\underline{R}}_{0}=\underline{0}$, la relation (6) se ramène banalement (au moins dans un domaine simplement connexe) au cas d'un matériau présentant un état naturel (5) en faisant un meilleur choix de la configuration de référence, soit $\underline{\underline{\varepsilon}}^{\prime}=\underline{\underline{\varepsilon}}-\underline{\varepsilon}_{0}$.

Si la déformation initiale n'est pas compatible, $\underline{\underline{R}}_{0} \neq \underline{\underline{0}}$, de nouvelles caractéristiques apparaissent : - il est impossible de réaliser la condition $\underline{\underline{\sigma}}(\underline{\mathrm{x}})=\underline{\underline{0}}$ dans un domaine matériel $\Omega$ de volume non nul : on aurait alors $\underline{\underline{\varepsilon}}=\underline{\underline{\varepsilon}}_{0}$ alors que $\underline{\underline{R}}\{\varepsilon\}=\underline{\underline{0}}$ (par définition) et $\underline{\underline{R}}_{0}\left\{\underline{\underline{\varepsilon}}_{0}\right\} \neq \underline{\underline{0}}$; - la définition de la configuration initiale présente un certain caractère arbitraire, en l'absence d'un état naturel. Si $\underline{\xi}^{\prime}$ décrit le déplacement depuis la configuration de référence utilisée pour écrire (6) jusqu'à une autre configuration, et si $\varepsilon^{\prime}$ est le tenseur de déformation associé, la loi de comportement (4) peut être réécrite : 


$$
\underline{\underline{\varepsilon}}-\underline{\underline{\varepsilon^{\prime}}}=\underline{\underline{\varepsilon}} 00-\underline{\underline{\varepsilon^{\prime}}}+\frac{1+v}{\mathrm{E}} \underline{\underline{\sigma}}-\frac{v}{\mathrm{E}}(\operatorname{tr} \underline{\underline{\sigma}}) \underline{\underline{1}}
$$

où $\underline{\varepsilon}-\underline{\varepsilon}^{\prime}$ est le nouveau tenseur de déformation, calculé poür la nouvelle configuration de référence. La définition de la déformation initiale change, elle devient $\underline{\underline{\varepsilon}}_{0}^{\prime}=$ $\underline{\varepsilon}_{0}-\underline{\varepsilon}^{\prime}$; en revanche le tenseur d'incompatibilité est invariant par changement de configuration de référence selon (4). En ce sens le tenseur d'incompatibilité $\underline{R}_{0}$ indépendant de toute configuration de référence, contient toute l'information nécessaire au calcul des contraintes résiduelles, comme il sera montré dans les paragraphes suivants.

\section{4}

\section{Calcul des contraintes résiduelles}

\section{1}

\section{Système vérifié par les contraintes}

Pour le matériau défini par la relation de comportement (6), la détermination des contraintes résiduelles s'effectue en résolvant le système suivant (7) obtenu en ajoutant au système (1) les conditions décrites précédemment portant sur les déplacements et déformations :

$$
\begin{aligned}
& \underline{\operatorname{div}} \underline{\underline{\sigma}}=\underline{0} \text { dans } \Omega \\
& \underline{\underline{\sigma}} . \underline{\mathrm{n}}=\underline{0} \operatorname{sur} \partial \Omega \\
& \underline{\underline{\varepsilon}}=\underline{\varepsilon}_{0}+\frac{1+v}{E} \underline{\underline{\sigma}}-\frac{v}{E}(\operatorname{tr} \underline{\underline{\sigma}}) 1 \\
& \underline{\underline{\mathrm{R}}}[\underline{\underline{\varepsilon}}]=\underline{\underline{0}} \text { dans } \Omega \\
& \text { Fermeture de } \xi \\
& \text { [[ } \underline{\underline{\sigma}}]] . \underline{N}=\underline{0} \operatorname{sur} \Gamma \\
& {[[\xi]]=\underline{0} \operatorname{sur} \Gamma}
\end{aligned}
$$

La quatrième condition traduit la compatibilité de $\underline{\varepsilon}=\underline{\varepsilon}(\mathrm{x})$; la cinquième condition ne vaut que pour un domaine $\Omega$ non simplement connexe ; $\Gamma$ désigne une éventuelle frontière interne de discontinuité de $\underline{\underline{\sigma}}$.

On s'efforce, dans l'esprit de la méthode de Beltrami-Michell (J. Salençon, 1995) de remplacer (7) par un système dans lequel n'intervient plus que l'inconnue $\underline{\sigma}$. En combinant les troisième et quatrième conditions de (7) il vient, en tenant compte de la première condition :

$\mathrm{ER}_{\underline{\underline{R}}}-(1+v) \Delta \underline{\underline{\sigma}}-\operatorname{grad} \operatorname{grad}(\operatorname{tr} \underline{\underline{\sigma}})+v \Delta(\operatorname{tr} \underline{\underline{\sigma}}) \underline{\underline{1}}=\underline{\underline{0}}$

On peut simplifier quelque peu l'expression précédente en prenant sa trace afin d'éliminer $\Delta(\operatorname{tr} \underline{\underline{\sigma}})$ :

$E \underline{\underline{R}}_{0}-(1+v) \Delta \underline{\underline{\sigma}}-\operatorname{grad} \underline{\operatorname{grad}}(\operatorname{tr} \underline{\underline{\sigma}})+v E R_{0} \underline{\underline{1}} /(2(1-v))=\underline{\underline{0}}(8)$ où l'on a posé $\mathrm{R}_{0}=\operatorname{tr} \underline{\underline{R}}_{0}$.

Il reste à établir que (8), avec la condition $\underline{\sigma} \cdot \underline{n}=\underline{0}$, équivaut à (7). En calculant la divergence de (8) et compte tenu de la relation $2 \operatorname{div} \underline{\underline{R}}_{0}=\underline{\operatorname{grad}} \mathrm{R}_{0}$ (voir 1.2.a), il vient :

$\Delta(\operatorname{div} \underline{\sigma})=\underline{0}$
Autrement dit, (8) implique que div $\underline{\underline{\sigma}}$ est une fonction harmonique; pour que cette fonction soit nulle dans $\Omega$ il suffit alors qu'elle le soit sur $\partial \Omega$ et on obtient le système (9) qui équivaut au sous-système constitué par les quatre première relations de (7):

(8) dans $\Omega$

$\underline{\underline{\sigma}} \cdot \underline{n}=\underline{0} \quad$ sur $\partial \Omega$

$\underline{\operatorname{div}} \underline{\underline{\sigma}}=0 \quad$ sur $\partial \Omega$

Déplacements et déformations n'apparaissent plus dans (9) mais on n'a tenu compte ni des conditions de fermeture, ni des conditions relatives à une surface de discontinuité $(\Gamma)$, qui apparaissent dans (7). Ces conditions sont discutées en annexe.

\section{2}

\section{Propriétés du système vérifiées par les contraintes}

L'examen du système (9) montre que les contraintes résiduelles sont proportionnelles au module élastique $E$ et ne dépendent par ailleurs que du domaine $\Omega$, du coefficient de Poisson $v$ et du tenseur d'incompatibilité $\underline{R}_{0}=\underline{R}_{0}(x)$; les déformations initiales $\underline{\varepsilon}_{0}=\underline{\varepsilon}_{0}(x)$ n'apparaissent plus que par l'intermédiaire de $\underline{\mathrm{R}}_{0}$; en particulier la configuration de référence pour le calcul des déformations n'apparaît plus dans (9).

\section{3}

\section{Exemples}

\section{Dilatation thermique}

On a déjà envisagé le cas des dilatations d'origine thermique, $\underline{\varepsilon}_{0}=\alpha \mathrm{T} \underline{\underline{1}}$; on a dans ce cas :

$$
\underline{\underline{R}}_{0}=-\Delta(\alpha \mathrm{T}) \underline{\underline{1}}-\underline{\underline{\operatorname{grad}}} \underline{\operatorname{grad}}(\alpha \mathrm{T})
$$

En particulier, une distribution affine de températures $\alpha \mathrm{T}=\underline{\mathrm{A}} \cdot \underline{\mathrm{x}}+\mathrm{B}$, n'engendre pas de contraintes résiduelles.

\section{2 \\ Cylindre}

On considère le cas d'un cylindre de hauteur $2 \mathrm{~h}$, rayon b, et on choisit un tenseur d'incompatibilité dont la forme particulière est :

$$
\begin{aligned}
& \mathrm{R}_{0}^{\mathrm{rr}}=\mathrm{R}_{0}^{\theta \theta}=(2-v) \mathrm{r}_{0} \\
& \mathrm{R}_{0}^{\mathrm{zz}}=-2 v \mathrm{r}_{0}
\end{aligned}
$$

où $v$ est le coefficient de Poisson et $r_{0}$ une constante. Dans ce cas particulier les contraintes résiduelles prennent la forme :

$$
\begin{aligned}
& \sigma_{\mathrm{rr}}=\operatorname{Er}_{0}\left(\mathrm{r}^{2}-\mathrm{b}^{2}\right) / 8 \\
& \sigma_{\theta \theta}=\operatorname{Er}_{0}\left(3 \mathrm{r}^{2}-\mathrm{b}^{2}\right) / 8 \\
& \sigma_{\mathrm{zz}}=0
\end{aligned}
$$


Cette solution ne dépend pas de l'élancement $\mathrm{h} / \mathrm{b}$; pour $r_{0}>0$ fixé, la contrainte sur l'axe de révolution $\sigma_{r}$ $(\mathrm{r}=0)=\sigma_{\theta \theta}(\mathrm{r}=0)=-\mathrm{Er}_{0} \mathrm{~b}^{2} / 8$ est une compression qui varie comme le carré du rayon du cylindre; la contrainte tangentielle sur la surface latérale est une traction $\sigma_{\theta \theta}(\mathrm{b})=\mathrm{Er}_{0} \mathrm{~b}^{2} / 4$.

Cet exemple (très particulier) suggère ce que pourrait être une méthode expérimentale de détermination du tenseur d'incompatibilité en Mécanique des Roches : en perçant un trou cylindrique coaxial de rayon a dans l'éprouvette cylindrique, on détermine un déplacement:

$u(r) / r=-E_{0} a^{2} b^{2}\left(\frac{1+v}{r^{2}}+\frac{1-v}{b^{2}}\right) / 8$ dont la mesure permet de déterminer $\mathrm{r}_{0}$.

\section{Contraintes résiduelles dans un cylindre de roches}

\section{1}

\section{Introduction}

Il est difficile d'imaginer que le tenseur d'incompatibilité $\underline{R}_{0}$ conserve un signe et une intensité constants dans un très grand volume continu de roches. Supposons en effet que $\underline{\underline{R}}_{0}$ soit de l'ordre de $\mathrm{r}_{0} \underline{1}$; les contraintes résiduelles sont alors de l'ordre de $\mathrm{E}_{\bar{r}} \ell^{2}$ si est la dimension du volume considéré : ces contraintes sont très grandes dans un volume de grande dimension de sorte qu'il faut supposer que $\underline{\underline{R}}_{0}$ varie en fait sensiblement à grande échelle, ou que le volume se fracture en blocs de plus petite dimension. En revanche, il est raisonnable de penser qu'à petite échelle (carotte) le tenseur d'incompatibilité $\underline{\underline{R}}_{0}=\underline{R}_{0}(\underline{x})$ est peu variable et qu'on peut au premier ordre $\overline{l e}$ considérer comme constant. En remontant la carotte à la surface, on supprime les contraintes d'origine gravitaire (compressives) et il ne subsiste plus que les contraintes résiduelles, qui sont des compressions et des tractions, puisqu'un champ d'autocontraintes vérifie la condition

$$
\int_{\Omega} \sigma \mathrm{d} \Omega=\underline{=}
$$

On sait que les roches résistent plus mal aux tractions qu'aux compressions ; il faut donc comparer à la résistance à la traction la quantité $\operatorname{Er}_{0} \ell^{2}$, où $\ell$ est la dimension de la carotte. Dans certains cas, si $r_{0}$ est ( grand », la résistance à la traction sera atteinte, la carotte se fractionnera en morceaux plus petits dans lesquels les contraintes résiduelles seront moins intenses.

\section{2}

\section{Cas d'un cylindre de roches}

On généralise le cas envisagé en 3.3.2. en considérant le cas d'un cylindre de roche, de rayon $b$, demihauteur h; ce cas est celui d'une éprouvette taillée dans une carotte prélevée à grande profondeur. La discussion qui suit suppose qu'on ait pu prélever et tailler l'éprouvette sans provoquer aucune sorte de dommage, hypothèse sans doute discutable pour un prélèvement réel; enfin, on admet que la loi :

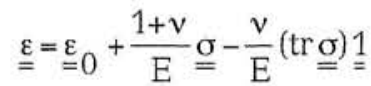

s'applique à l'ensemble du trajet de contraintes subi par le matériau depuis sa situation initiale dans le massif, avant prélèvement, jusqu'à sa situation finale non chargée au laboratoire.

On se préoccupe des contraintes résiduelles dans le cylindre. On sait que ces contraintes sont entièrement déterminées par le tenseur d'incompatibilité du matériau (et par les dimensions h et b de l'éprouvette, ainsi que par ses paramètres élastiques $\mathrm{E}$ et $\mathrm{v}$ ).

On suppose que ce tenseur est constant dans l'éprouvette. On suppose de plus - mais ce sera souvent une hypothèse plus discutable que la précédente que le tenseur d'incompatibilité a les mêmes symétries que l'éprouvette, dont l'axe de révolution était à peu près vertical avant son prélèvement par carottage. Les seules composantes non nulles sont alors :

$$
\mathrm{R}_{0}^{\mathrm{rr}}=\mathrm{R}_{0}^{\theta \theta}=\alpha \quad \mathrm{R}_{0}^{\mathrm{ZZ}}=\beta
$$

et la solution générale du problème prend la forme :

$$
\underline{\sigma}=E b^{2}[\alpha \underline{\underline{\varphi}} \alpha \underline{x}(\underline{b}, h / b, v)+\beta \underline{\underline{\varphi}} \beta \underline{\beta}(\underline{x} / b, h / b, v)]
$$

où $\varphi_{\alpha}$ et $\varphi_{\beta}$ sont deux fonctions à déterminer numériquement.

Le traitement numérique du problème a été effectué à l'aide du logiciel VIPLEF écrit par M. Tijani au Centre de géotechnique et d'exploitation du sous-sol de l'École des mines de Paris. La formule (11) montre que le choix du module E équivaut au choix de l'unité de contraintes. Le coefficient de Poisson a été pris égale à $v=0,25$ et dans les cas représentés l'élancement est égal à $\mathrm{h} / \mathrm{b}=2$. Le paragraphe précédent montre qu'on dispose d'un cas test, correspondant à $\alpha / \beta=-(2-v) /(2 v)=-3,5$ (pour $v=0,25$ ), pour lequel la distribution des contraintes est connue explicitement. Le calcul de ce cas test a montré que la solution numérique obtenue en adoptant pour un quart de section méridienne un maillage de quadrilatères à 9 nœuds (515 nœuds au total) est en excellent accord avec la solution explicite.

La solution générale est une combinaison linéaire du ( $\operatorname{cas} \alpha$ ) associé à $\beta=0$ et $\alpha=1 \mathrm{~m}^{-2}$ et du ( $\operatorname{cas} \beta$ ) associé à $\alpha=0$ et $\beta=1 \mathrm{~m}^{-2}$ respectivement. Ces deux cas sont représentés sur les figures 1 et 2 qui donnent les composantes $\sigma_{r \mathrm{r}}, \sigma_{\theta \theta^{\prime}} \sigma_{z z}$ et $\sigma_{\mathrm{rr}}$ du tenseur des contraintes, rapportees respectivement à E $\alpha b^{2}$ ou $E \beta b^{2}$.

\section{3}

\section{«Cas $\alpha$ »}

La distribution des contraintes radiales $\sigma_{\mathrm{rr}}=\sigma_{\mathrm{rr}}(\mathrm{r}, \mathrm{z})$ n'est pas très différente de celle du " cas test » $\alpha=2-v, \beta$ $=-2 v$, au signe près : la contrainte radiale croît (en valeur absolue) depuis la périphérie (elle est contrainte principale et égale à zéro en $\mathrm{r}=\mathrm{b}$ ) vers le centre, où elle est pratiquement constante dans le (c cœur » de rayon $\mathrm{b} / 3$ environ; elle ne dépend pratiquement que du rayon, comme dans le cas test, à l'exception notable du 

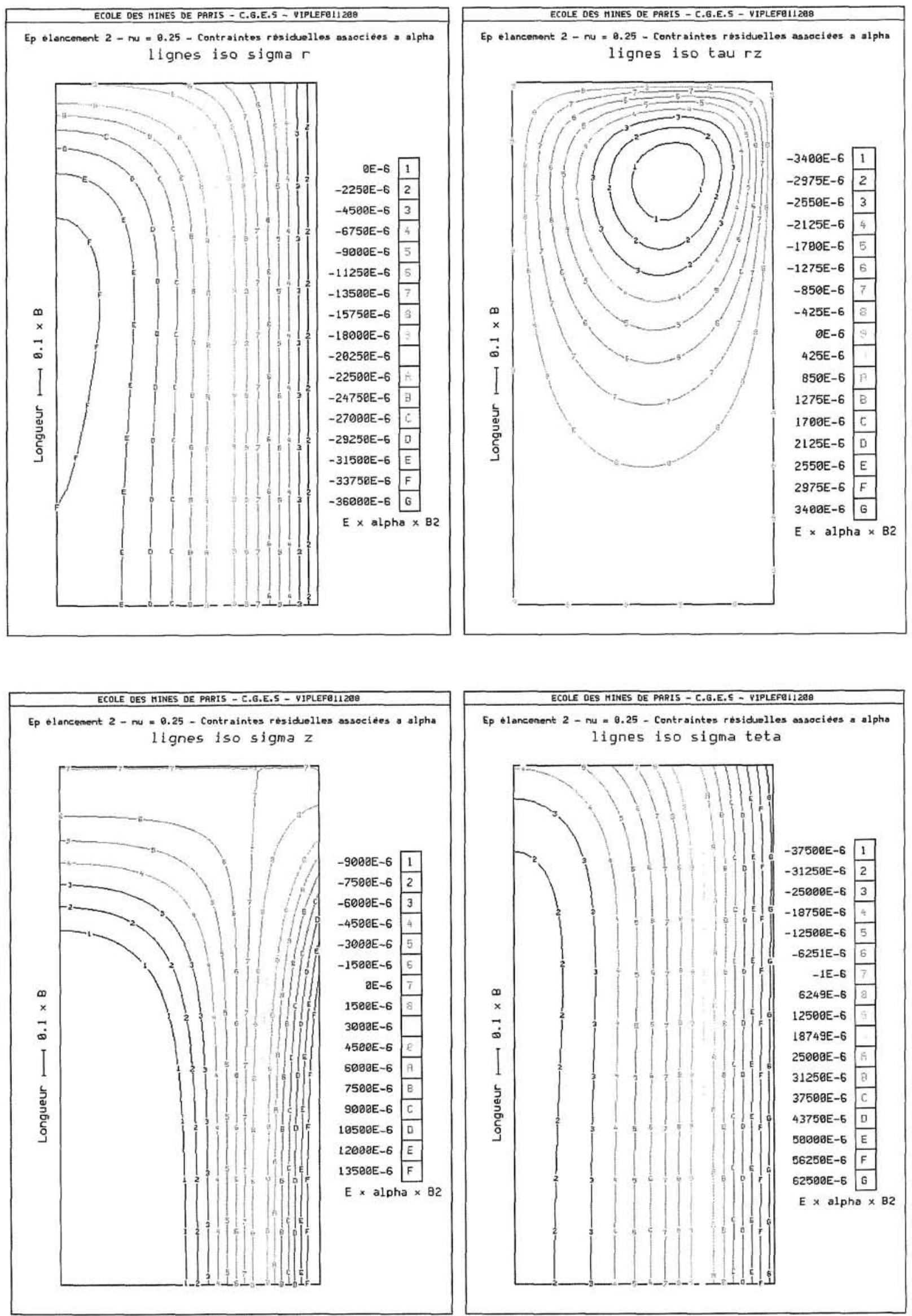

FIG. 1. Lignes iso-contraintes $\sigma_{\mathrm{rr}}, \sigma_{\mathrm{rz}}$ et $\sigma_{\theta 9}$ dans un quart de section méridienne dans le cas $\alpha=1 \mathrm{~m}^{-2}$, $\beta=0$. L'unité de contrainte est $E \alpha b^{2}$, b est le rayon du cylindre.

Iso-stress lines, $\sigma_{r}, \sigma_{r z}$ et $\sigma_{\theta \theta}$ respectively in the upper right side of a vertical cross section in the case $\alpha=1 \mathrm{~m}^{-2}, \beta=0$. The stress unit is E $\alpha b^{2}, b$ is the cylinder radius. 

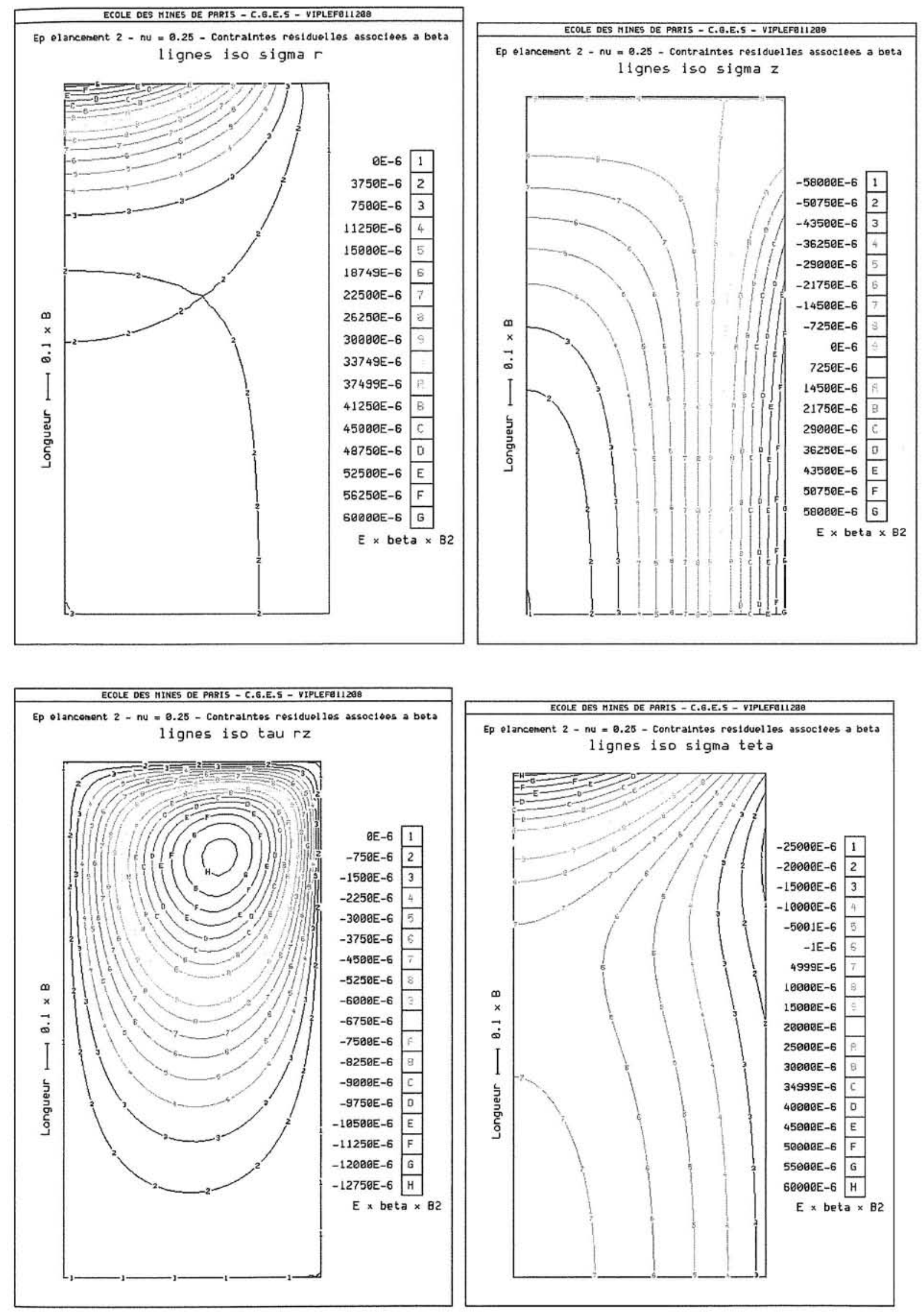

FIG. 2. Lignes iso-contraintes dans le cas $\alpha=0, \beta=1 \mathrm{~m}^{-2}$. Iso-stress lines in the case $\alpha=0, \beta=1 \mathrm{~m}^{-2}$. 
voisinage des faces où la décroissance (en valeur absolue) de la contrainte radiale quand on s'approche de l'axe du cylindre est moins rapide que dans les sections horizontales moins proches des faces.

On peut faire une remarque analogue pour les contraintes tangentielles (principales) $\sigma_{\theta \theta}=\sigma_{\theta \theta}(\mathrm{r}, \mathrm{z})$ : ce sont des tractions à la périphérie, qui se réduisent progressivement quand on s'éloigne de la face latérale et deviennent des compressions quand on s'approche de l'axe du cylindre.

Le point le plus remarquable réside dans l'apparition de contraintes axiales qui sont des tractions à la périphérie et des compressions au cœur de l'éprouvette. Toutefois, les tractions axiales à la périphérie sont plus faibles, d'un facteur 4 environ, que les tractions tangentielles à la périphérie. Dans le cas $\alpha$ on peut attendre pour de grandes valeurs de E $\alpha b^{2}$ (grandes en comparaison de la résistance à la traction) l'apparition de fissures axiales à la périphérie de l'éprouvette.

Parmi l'infinité de champs de déformations résiduelle $\underline{\varepsilon}_{0}=\underline{\varepsilon}_{0}(\mathrm{r}, \mathrm{z})$ dont le tenseur d'incompatibilité est celui du ( $\operatorname{cas} \alpha)$, on a le champ sphérique :

$$
\varepsilon_{0}^{\mathrm{rr}}=\varepsilon_{0}^{\theta \theta}=\varepsilon_{0}^{\mathrm{zZ}}=\alpha\left(\mathrm{z}^{2}-\mathrm{r}^{2}\right) / 4
$$

que l'on peut interpréter comme résultant d'un écart de température.

On a également fait le calcul pour un élancement $\mathrm{h} / \mathrm{b}=5$ au lieu de $\mathrm{h} / \mathrm{b}=2$ : les contraintes $\sigma_{\mathrm{rr}}, \sigma_{\theta \theta^{\prime}} \sigma_{z z}$ sont pratiquement principales sur les trois quarts de la hauteur de l'éprouvette, avec une distribution très proche de celle du ( cas test ): ce n'est qu'au voisinage des faces supérieure et inférieure que la solution s'écarte de celle du cas test ; les contraintes dans ces régions ont la même allure que dans le cas d'un élancement 2

\section{4}

\section{"Cas $\beta$ »}

La distribution des contraintes radiales $\sigma_{\mathrm{rr}}=\sigma_{\mathrm{rr}}$ $(\mathrm{r}, \mathrm{z})$ est cette fois très distincte de celle du « cas test ». Pour effectuer des comparaisons avec le cas précédent (cas $\alpha$ ) on prend comme contrainte de référence la contrainte tangentielle qui régnait à la périphérie de l'éprouvette, soit environ $\sigma_{\theta \theta}(b, 0)=36$. $10^{-3} \mathrm{E} \alpha \mathrm{b}^{2}$.

Les contraintes radiales, dans le cas $\beta$, sont des tractions qui, dans la majeure partie de l'éprouvette, sont inférieures au dixième de la contrainte de référence. Elles n'approchent la valeur de la contrainte de référence que dans la partie centrale des faces supérieure et inférieure, et sur une faible épaisseur.

Les contraintes tangentielles (qui sont principales) $\sigma_{\theta \theta}=\sigma_{\theta \theta}(\mathrm{r}, \mathrm{z})$ montrent, à mi-hauteur de l'éprouvette, une succession compression périphérique-traction centrale, mais avec des valeurs absolues modérées, de l'ordre du quart de la contrainte de référence pour la valeur absolue de la compression périphérique, et du huitième de la contrainte de référence pour la traction centrale ; en revanche, comme pour les contraintes radiales, des tractions importantes, de l'ordre de la contrainte de référence, se développent au centre des faces inférieure et supérieure.

La contrainte axiale $\sigma_{z z}=\sigma_{z z}(\mathrm{r}, \mathrm{z})$ est une forte compression au cœur de l'éprouvette et une traction d'intensité comparable à la périphérie, à mi-hauteur de l'éprouvette. Les lignes $\sigma_{z z}=0$ coïncident, dans les cas $\alpha$ et $\beta$, puisque le ( cas test ), dans lequel $\sigma_{z z}=0$ dans toute l'éprouvette, est une combinaison linéaire de ces deux cas.

On peut interpréter le cas $\beta$ comme résultant d'une déformation initiale associée à un écart de température ;

$$
\varepsilon_{0}^{r r}=\varepsilon_{0}^{\theta \theta}=\varepsilon_{0}^{z z}=\beta\left(r^{2}-3 z^{2}\right) / 8
$$

On peut attendre pour de grandes valeurs de $E \beta b^{2}$ l'apparition d'une fissuration au centre des faces supérieure et inférieure et des fissures perpendiculaires à l'axe de révolution sur la surface latérale de l'éprouvette.

\section{Conclusion}

On a montré que les caractéristiques « résiduelles ») d'un matériau étaient tout entières décrites par le tenseur d'incompatibilité, ou tenseur de Ricci des « déformations initiales ). Ces dernières sont moins aptes à décrire ces caractéristiques car elles dépendent du choix, souvent arbitraire, de la configuration de référence.

En particulier le champ de tenseur d'incompatibilité permet en général de déterminer l'état de contraintes résiduelles dans une éprouvette. En supposant que ce tenseur est constant dans une éprouvette de petite taille, on peut esquisser une typologie des champs de contraintes résiduelles. Le cas d'un champ purement axial permet de prévoir des tractions importantes à la périphérie du cylindre et au centre des faces supérieure et inférieure.

L'étude présentée permet de fournir un cadre d'interprétation pour une détermination expérimentale du tenseur d'incompatibilité dans une éprouvette, qui pourrait reposer sur la mesure des déplacements induits par le percement d'un trou cylindrique coaxial.

\footnotetext{
REMERCIEMENTS

Les auteurs remercient A. Bioget, M. Bonnet, J. Gaombalet RM. Pradeilles-Duval, C. Stolz, O. Simionescu, M. Tijani, A. Zaou pour les discussions éclairantes qu'ils ont permises. $O$. Simionescu a écrit à cette occasion une formulation intégrale du problème de la détermination des contraintes résiduelles.
} 


\section{Annexe}

Conditions de fermeture et conditions sur une surface de discontinuité

On a vu que le système (9) permet en principe de calculer les contraintes résiduelles (ou autocontraintes) dans un domaine $\Omega$ lorsque $E, v$ et $\underline{R}_{0}$ sont donnés. En fait ce système n'est pas complet dans certains cas, que l'on examinera à travers deux exemples.

\section{Conditions de fermeture}

On considère un tube de rayons a et b, $\log (b / a)<$ $2 / 3$, réalisé dans un matériau élastoplastique de Tresca ; la cohésion est notée $\mathrm{C}$. On fait l'hypothèse des déformations planes; la pression appliquée sur la surface latérale extérieure est nulle, $\sigma_{r}(\mathrm{~b})=0$; la pression intérieure est croissante, $\sigma_{r r}(\mathrm{a})=-\mathrm{P}_{\mathrm{i}}$, jusqu'à atteindre l'écoulement libre soit $\mathrm{P}_{\mathrm{i}}=2 \mathrm{C} \log (\mathrm{b} / \mathrm{a})$; puis on décharge la structure soit $\mathrm{P}_{i}=0$. Quel est alors le champ de contrainte résiduelles ? A l'instant où l'écoulement libre est atteint, on a $\sigma_{p}^{\mathrm{rr}}=-2 \mathrm{CLog}(\mathrm{b} / \mathrm{r}), \sigma_{\mathrm{p}}^{\theta \theta}=2 \mathrm{C}+\sigma_{\mathrm{p}^{\prime}}^{\mathrm{rr}}$ la déformation plastique s'écrit :

$$
\varepsilon_{0}^{\mathrm{rr}}=-\varepsilon_{0}^{\theta \theta}=-\frac{2 \mathrm{C}}{\mathrm{E}}\left(1-v^{2}\right)\left(\frac{\mathrm{b}^{2}}{\mathrm{r}^{2}}-1\right)
$$

et il est facile de vérifier que le tenseur d'incompatibilité associé est $\underline{R}_{0}=\underline{0}$. Le système (9) admet alors la solution $\underline{\underline{\sigma}}=\underline{0}$, de sorte qu'on est tenté d'en déduire qu'il n'y a pas de contrainte résiduelle après déchargement de la structure.

Bien entendu ce résultat est incorrect. La solution correcte est obtenue en ajoutant aux contraintes $\sigma_{p}^{r r} \sigma_{p}^{\theta \theta}$ les variations de contrainte associées à la décharge de la structure par la loi incrémentale élastique de comportement :

$\sigma^{T r}=-2 \mathrm{C} \log (b / r)+2 C[\log (b / a)]\left(b^{2} / r^{2}-1\right) /\left(b^{2} / a^{2}-1\right) ;$ $\left.\sigma^{\theta \theta}=2 C-2 C \log (b / r)\right]-2 C\left[\log (b / a)\left(b^{2} / r^{2}+1\right) /\left(b^{2} / a^{2}-1\right)\right.$ et ces contraintes ne sont pas nulles.

On peut s'apercevoir d'une autre manière que la solution $\underline{\sigma}=\underline{0}$ ne convient pas pour l'état déchargé. Elle impliquerait que les variations des contraintes radiales et tangentielles entre l'état d'écoulement libre et l'état déchargé soient égales à $-\sigma_{p^{\prime}}^{r r}-\sigma_{p}^{\theta \theta}$ respectivement. On peut certes associer à ces variations de contraintes un déplacement en utilisant la loi incrémentale élastique de comportement ( $c^{\prime}$ est une conséquence de la propriété $\underline{\underline{R}}_{0}=\underline{\underline{0}}$ ). Mais ce déplacement prend la forme : $E \underline{\xi}=2\left(1-v^{2}\right) C\left[\left(r+\underline{x}^{2}\right) \underline{e}_{r}-2 r \sigma \underline{e}_{0}\right]$.

Ce déplacement n'est pas univoque et ne vérifie donc pas la condition de fermeture. En revanche, si on réalise une coupure ( radiale ) du cylindre, la solution $\underline{\underline{\sigma}}=\underline{\underline{0}}$ convient pour la structure déchargée. Cet exemple montre que, pour un domaine non connexe, la distribution des contraintes résiduelles dépend de l'histoire du chargement antérieur.

Il faut noter que dans le cas d'une sphère creuse (qui est un domaine simplement connexe) le tenseur d'incompatibilité associé aux déformations plastiques à l'écoulement libre n'est pas nul, de sorte que les contraintes résiduelles (après déchargement) ne sont pas supprimées même en (c coupant) la sphère.

\section{Conditions sur une surface de discontinuité}

On reprend le problème précédent en arrêtant le chargement lorsqu'une zone plastique de rayon $\mathrm{x}, \mathrm{a}<\mathrm{x}$ $<\mathrm{b}$, s'est développée ; on ramène alors la pression de $\mathrm{P}_{\mathrm{i}}=2 \mathrm{C} \log (\mathrm{x} / \mathrm{a})+\mathrm{C}\left(1-\mathrm{b}^{2} / \mathrm{x}^{2}\right)$ à $\mathrm{P}_{\mathrm{i}}=0$ et on réalise une coupure pour éviter les problèmes de ( fermeture ») évoqués plus haut. La déformation plastique s'écrit :

$$
\begin{gathered}
\varepsilon_{0}^{r r}=-\varepsilon_{0}^{\theta \theta}=-\frac{2 C}{E}\left(1-v^{2}\right)\left(\frac{x^{2}}{r^{2}}-1\right) \text { pour } a \leq r \leq x \\
\varepsilon_{0}^{r r}=-\varepsilon_{0}^{\theta \theta}=0 \text { pour } x \leq r \leq b
\end{gathered}
$$

Pour chacune des deux zones considérées on a $\underline{\underline{R}}_{0}=\underline{\underline{0}}$. Le champ de contraintes $\underline{\underline{\sigma}}=\underline{\underline{0}}$ vérifie dont, séparement dans chaque zone, les équations du système (9) et vérifie de plus la condition :

$$
\text { [[미] . } \underline{N}=0 \text { sur } \Gamma
$$

du système (7) sur la frontière $r=x$. Ce champ de contraintes n'est pas pour autant le champ de contraintes résiduelles dans ce problème. En effet on a de plus une condition pour le déplacement dans le passage de l'état chargé $\mathrm{P}_{\mathrm{i}}=2 \mathrm{C} \log (\mathrm{x} / \mathrm{a})+\mathrm{C}\left(1-\mathrm{x}^{2} / \mathrm{b}^{2}\right)$ à l'état déchargé $\mathrm{P}_{\mathrm{i}}=0$ :

$$
[[\xi]]=0 \operatorname{sur} \Gamma
$$

Si on note $\left(\underline{t}_{1}, \underline{t}_{2}, \underline{N}\right)$ une base orthonormée au point courant de la surface de discontinuité $\Gamma$, la condition précédente implique les conditions de Hadamard:

$$
[[\nabla \underline{\xi}]] \cdot \underline{t}_{i}=0 \quad i=(1,2)
$$

Ces six conditions scalaires se décomposent en quatre conditions relatives au plan tangent :

$$
2\left[\left[\varepsilon_{\mathrm{ij}}\right]\right]=\underline{\mathrm{t}}_{\mathrm{i}} \cdot\left[\left[\underline{\underline{\nabla \zeta}}+{ }^{\tau} \underline{\underline{\nabla \zeta}}\right]\right] \cdot \underline{\mathrm{t}}_{\mathrm{j}}=0
$$

qui permettent, avec (12), d'écrire des conditions de saut portant sur les contraintes seulement; mais subsistent deux conditions :

$$
\underline{N} \cdot[[\nabla \underline{\xi}]] \cdot \underline{t}_{-1}=0
$$

qui portent sur le déplacement de sorte que le système (9) (12) (13) n'est pas suffisant pour déterminer le champ de contraintes résiduelles.

Autrement dit, lorsqu'un corps est composé de parties distinctes par leurs propriétés mécaniques ( $E, v)$ ou par l'histoire des évolutions irréversibles subies, les conditions ( d'assemblage ») de ces diverses parties doivent être précisées.

Dans les deux cas présentés dans cette annexe, on remarque qu'il existe un état naturel pour toute partie suffisamment petite de la structure après sa plastification complète ou partielle : il suffit d'extraire de la structure une partie connexe (pour le premier exemple) ou une partie qui n'est pas traversée par la frontière élastoplastique (pour le second exemple). 
Archer R.R. - Growth Stresses and Strains in Trees. Springer Series in Wood Sciences. E. Timell Springer Verlag, Berlin, 1986.

Ballard P., Constantinescu A. - On the inversion of subsurface residual stresses from surface stress measurements. $J$. Mech. Phys. Solids, vol. 42, n 11, 1994, p. 1767-1987.
Cornet F.H. - Stresses in Rock and Rock Masses. Comprehensive Rock Engineering, J.A. Hudson, ed., vol. 3, 1993, p. 297-327

Hardy H.R., Mangolds A. - Investigation of Residual Stresses in Salt. 5th Symp. on Salt, A.H. Coogen and L. Hauber ed. The Northern Ohio Geological Society, vol.1, 1980, p. 55-64.
Salencon J. - Mécanique du continu, vol. II. Ellipses, Paris, Coll. « Universités francophones », 1995. 